\title{
Tratamento da forma neovascular de degeneração macular relacionada à idade com drogas antiangiogênicas
}

\author{
Treatmentof neovascularage-related maculardegeneration with \\ antiangiogenicdrugs
}

\author{
Eduardo Büchele Rodrigues ${ }^{1}$ \\ Eglas Emanuel Rossi ${ }^{2}$ \\ Astor Grumann Junior ${ }^{3}$ \\ Carsten H. Meyer ${ }^{4}$ \\ Allen C. Ho
}

Departamento de Retina, Serviço de Oftalmologia, Hospital Regional São José, Instituto de Olhos Florianópolis - Centro Oftalmológico - Florianópolis (SC) - Brasil. ${ }^{2}$ Departamento de Retina, Serviço de Oftalmologia, Hospital Regional São José, Instituto de Olhos Florianópolis - Centro Oftalmológico - Florianópolis (SC) - Brasil. ${ }^{3}$ Departamento de Retina, Serviço de Oftalmologia, Hospital Regional São José, Instituto de Olhos Florianópolis - Centro Oftalmológico - Florianópolis (SC) - Brasil. ${ }^{4}$ Departamento de Oftalmologia, Hospital de Olhos, Phillips-University, Marburg, Alemanha.

${ }^{5}$ Retina Service, Wills Eye Hospital, Philadelphia (PA) - USA.

Endereço para correspondência: Eduardo B. Rodrigues. Rua Presidente Coutinho, 579 - Cj. 501 - Florianópolis (SC) CEP 88015-000

E-mail: edubrodriguess@yahoo.com.br

Recebido para publicação em 11.10.2005

Última versão recebida em 26.12.2005

Aprovação em 10.02.2006

Fontes de auxílio à pesquisa: Enquanto na preparação deste artigo Eduardo $\mathrm{B}$. Rodrigues recebeu apoio à pesquisa de três Instituições da Alemanha e Estados Unidos: DAAD, Fehr Foundation, e Eye Tumor Research Foundation.

Nota Editorial: Depois de concluída a análise do artigo sob sigilo editorial e com a anuência do Dr. Eduardo Cunha de Souza sobre a divulgação de seu nome como revisor, agradecemos sua participação neste processo.

\begin{tabular}{|l|}
\hline RESUMO \\
\hline Degeneração macular relacionada à idade (DRMI) é a principal causa de \\
cegueira no mundo ocidental. Várias formas clínicas foram reconhecidas, \\
e membrana neovascular coroideana (MNSR) representa manifestação \\
importante passível de tratamento. O tratamento de MNSR tem sido um \\
foco importante de pesquisa nas últimas décadas e a primeira terapia \\
estabelecida baseada em evidência foi a fotocoagulação a laser, que \\
reduziu o risco de perda visual em lesões extrafoveais. No fim da década \\
de 90 a terapia fotodinâmica foi estabelecida como método eficiente de \\
tratamento de MNSR predominantemente clássicas e ocultas. Terapias \\
adicionais como a translocação macular, cirurgia submacular, e protrom- \\
bose mediada por indocianina verde estão atualmente em investigação \\
em ensaios clínicos em larga escala. A biologia molecular permitiu \\
recentemente uma melhor compreensão da patogênese da DMRI e o fator \\
de crescimento vascular endotelial foi reconhecido como um mediador- \\
chave na angiogênese da formação de MNSR. Portanto, a abordagem \\
farmacológica surge como opção terapêutica no tratamento da MNSR. O \\
primeiro agente terapêutico aprovado pelo FDA é o aptâmero pegaptanib \\
sódio(Macugen $\left.{ }^{\circledR}\right)$, que inativa a isoforma fundamental para a angiogênese \\
intra-ocular: VEGF ${ }_{165}$ Outros inativadores de VEGF como ranibizumab \\
RhuFab V2 (Lucentis $\left.{ }^{\circledR}\right)$ e bevacizumab (Avastin $\left.{ }^{\circledR}\right)$ estão em avaliação em \\
estudos clínicos. Resultados impressionantes de bevacizumab intravítreo \\
foram liberados recentemente. Adicionalmente, o derivado de esteróides \\
acetato de anecortave, assim como o corticosteróide acetato de trianci- \\
nolona têm sido propostos como métodos no tratamento de DMRI- \\
neovascular. Este artigo apresenta os princípios e resultados iniciais na \\
terapia antiangiogênica farmacológica da MNSR na DMRI. \\
\hline
\end{tabular}

Descritores: Degeneração macular, Neovascularização retiniana; Fator de crescimento do endotélio vascular; Inibidores de angiogênese

\section{INTRODUÇÃO}

Degeneração macular relacionada à idade (DMRI) é a principal causa de cegueira legal em pacientes acima de 60 anos no mundo ocidental ${ }^{(1-4)}$. Os fatores de risco estabelecidos para a doença são: idade avançada, etnia caucasiana, fumo, e hipertensão arterial sistêmica. A forma neovascular da DMRI é caracterizada por um crescimento de vasos sanguíneos no espaço sub-retiniano, denominada membrana neovascular sub-retiniana (MNSR) ${ }^{(5-6)}$. A MNSR estende-se anteriormente através de um defeito na membrana de Bruch para o espaço abaixo do epitélio pigmentar da retina (EPR), e/ou da 
retina neurosensorial, levando ao acúmulo de líquido no espaço sub-retiniano, sangue, ou lipídios. Estes efeitos secundários da MNSR, adicionados à presença de um tecido cicatricial ou fibroso, ocasiona a perda da visão central. O tratamento da DMRI na sua forma exsudativa representa um dos maiores desafios na terapêutica oftalmológica atual. Os tratamentos disponíveis até anos recentes incluem: fotocoagulação a laser, terapia fotodinâmica (PDT), cirurgia submacular, translocação macular, protrombose mediada por indocianina verde (iMP), e suplementos vitamínicos ${ }^{(7-9)}$. Uma nova abordagem do tratamento do MNSR, a classe de drogas antiangiogênicas, foi resultado de uma melhor compreensão da patobiologia da DMRI. Várias drogas encontram-se em fase avançada de investigação ou já disponíveis para o uso clínico e podem ser divididas em dois grandes grupos. Enquanto em um grupo estão aquelas inibidoras de fator de crescimento vascular endotelial (VEGF): pegaptanib sódio, ranibizumab, "VEGFtrap", e siRNA; no outro grupo encontram-se agentes antiangiogênicos com propriedades corticosteróides: squalamina, acetato de anecortave, e o corticosteróide acetato de triancinolona (AT). Este artigo objetiva revisar e discutir os princípios farmacológicos, mecanismos de ação, e a aplicação clínica destas novas drogas antiangiogênicas para o tratamento da DMRI-neovascular.

\section{Patogênese da membrana vascular sub-retiniana: o papel chave do VEGF}

Em 1876 Sattler descreveu pioneiramente a presença de vasos sanguíneos entre o EPR e a membrana de Bruch $^{(10)}$. Desde então investigação baseada em histologia de autópsia de pacientes com DMRI, de MNSR removidas cirurgicamente, testes in vitro, e experimentos in vivo em animais contribuíram para o entendimento da fisiopatogenia biomolecular da $\mathrm{DMRI}^{(11-14)}$. As várias hipóteses propostas como causas da DMRI neovascular envolvem múltiplos fatores como: 1) vascular / isquêmica; 2) senescência; 3) inflamatórios; 4) estresse oxidativos; 5) genéticos (Figura 1). A conjunção destes diferentes mecanismos na retina promovem um desequilíbrio de mediadores angiogênicos, peptídeos, citocinas e proteínas para o lado da produção de fatores angiogênicos ${ }^{(15-19)}$. Na fase inicial, o EPR e os fotorreceptores retinianos produzem principalmente VEGF, mas também a proteína de colonização monocítica (MCP-1) e interleucina 8 (IL-8) ${ }^{(20)}$. Os macrófagos são então atraídos da coriocapilar para a membrana de Bruch. Os macrófagos expressam fator de necrose tumoral $\alpha$ (TNF- $\alpha$ ), que estimula a produção pelo EPR de IL8, MCP-1 e VEGF, assim como a migração do EPR. Paralelamente o VEGF induz a proliferação e migração de células vasculares endoteliais. Neste momento há a formação da MNSR em si e seu crescimento em uma fase inflamatória ativa. Em certo momento, MNSR estabiliza-se e há um equilíbrio entre os fatores próangiogênicos como MMP, VEGF, angiopoentinas 1,2 e plasminogênio/fibrina com os fatores antiangiogênicos naturais como PEDF e TIMP. A conseqüente predominância de citoci- nas anti-proteolíticas, anti-migratórias e antiangiogênicas resulta em uma fase involucional da MNSR, clinicamente a cicatriz disciforme.

Em 1983, um peptídeo de sequiência de aminoácidos até então desconhecida foi isolado de líquido em porcos com ascite $^{(21)}$. Ele foi denominado inicialmente de fator de permeabilidade vascular por sua característica de permeabilidade vascular 50.000 vezes maior que a histamina. O descobrimento de propriedades como estimulação potente da neovascularização e do crescimento celular endotelial motivou a comunidade científica a redenominar esta substância de fator de crescimento vascular endotelial (vascular endotelial growth factor - VEGF) ${ }^{(22-23)}$. A família dos polipeptídios VEGF é composta pelos subgrupos VEGF-A, VEGF-B, VEGF-C, VEGF-D, VEGF-E, e o fator de crescimento placentário (placental growth factor - PIGF). O membro dessa família de VEGF mais extensivamente estudado até o momento constitui-se o VEGFA, que representa uma proteína de aproximadamente $40 \mathrm{kDa}$ envolvida no aumento da permeabilidade vascular e na angiogênese. VEGF-A age em vários componentes da angiogênese como proliferação e migração de células endoteliais, mecanismo de sobrevivência e migração celular endotelial, e o aumento da permeabilidade vascular ${ }^{(24-25)}$. Há até o presente seis isoformas conhecidas de VEGF-A em humanos: VEGF ${ }_{121}$, $\mathrm{VEGF}_{145}, \mathrm{VEGF}_{165}, \mathrm{VEGF}_{183}, \mathrm{VEGF}_{189}, \mathrm{VEGF}_{206}$. A isoforma predominante no olho é a $\mathrm{VEGF}_{165}$. As várias isoformas de VEGF interagem com os três membros de receptores de VEGF identificados: VEGFR-1 e VEGFR-2 e VEGFR-3 ${ }^{(22)}$. Paradoxicalmente, o VEGF e suas isoformas são necessários para a função normal de vários tecidos humanos. Por exemplo, a sua ausência no desenvolvimento vascular embrionário é letal para o feto, e a isoforma VEGF-A também foi demonstrada ter um efeito neuroprotetor direto em neurônios in vitro após lesão isquêmica ${ }^{(26)}$. Na neovascularização ocular, níveis aumentados de VEGF promoveram o surgimento de neovascularização

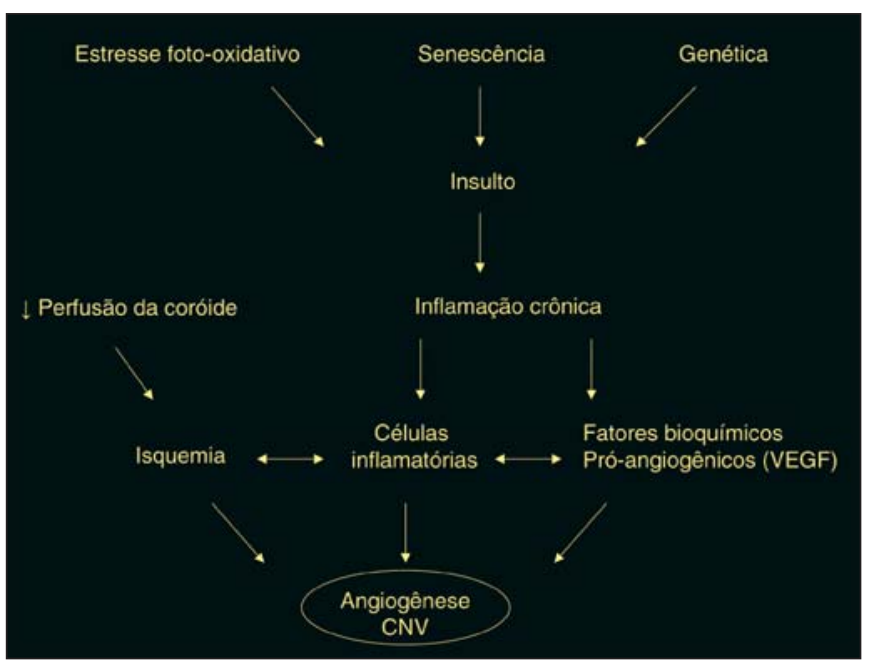

Figura 1 - Representação esquemática da fisiopatogenia da degeneração macular relacionada à idade 
na íris e na retina em modelos experimentais ${ }^{(27)}$, e esse peptídeo também tem sido associado à neovascularização ocular em doenças como retinopatia diabética, oclusões venosas retinianas, e retinopatia da prematuridade ${ }^{(28)}$. Quanto à forma exsudativa da DMRI, uma alta expressão de VEGF foi relatada em olhos de autópsia de DMRI e em MNSR removidas cirurgicamente. Adicionalmente, todos os três tipos receptores VEGFR já foram localizados no lado do endotélio da coriocapilar no sentido do $\mathrm{EPR}^{(29)}$. A inibição de VEGF em vários modelos animais resultou em regressão de neovascularização retiniana e coroideana. VEGF $_{165}$ foi necessário e suficiente para angiogênese da MNSR em modelos animais. A conjunção dessas evidências científicas sugere um papel fundamental do VEGF na patogênese da MNSR na DMRI, e transforma o VEGF em um importante alvo para drogas antiangiogênicas na terapia da DMRI-neovascular.

\section{Pegaptanib sódio (Macugen ${ }^{\circledR}$ )}

A melhor compreensão da patogênese biomolecular da DMRI neovascular e VEGF permitiu o desenvolvimento de terapias medicamentosas, sendo o pegaptanib sódio a primeira dessas drogas. Pegaptanib sódio (Macugen ${ }^{\circledR}$, Eyetech Pharmaceuticals, Inc., New York, NY) constitui em um aptâmero (do latim aptus significa "cabe" e merus "parte" ou "região") de acido ribonucléico com 28 bases nitrogenadas ligadas covalentemente a duas moléculas de polietileno glicosilado de 20 kDa. Aptâmeros são moléculas de DNA ou RNA que agem como anticorpos, apesar de não serem, desenvolvidas a partir de uma tecnologia chamada "Selex" (sigla em inglês para "Sistematic Evolution of Ligant Bioexplanation Enrichement"). Esse método consiste na seleção de uma seqüência de oligonucleotídeos com forma tridimensional a partir de uma seqüência única de centenas de moléculas de ácido nucléico ${ }^{(30)}$. A partir desse método é possível a geração de ácidos nucléicos que se ligam com alta afinidade a um particular alvo molecular. O aptâmero pegaptanib sódio ligase e bloqueia especificamente a atividade da isoforma $\mathrm{VEGF}_{165}$, seletividade essa que provém da formatação tridimensional do aptâmero. Com o objetivo de prolongar a atividade farmacocinética intra-ocular do aptâmero, a estrutura de seu carboidrato foi modificada para prevenir a degradação por enzimas endógenas como endonucleases e exonucleases, e uma molécula de polietileno glicosilado foi adicionada para aumentar a meiavida da droga no vítreo ${ }^{(31)}$ (Tabela 1).

O bloqueio da interação da isoforma VEGF $_{165}$ com seu receptor na célula endotelial pela droga pegaptanib inibiu a permeabilidade vascular e neovascularização retiniana em modelos animais ${ }^{(31)}$. Os ensaios clínicos fase 1 e 2 com injeção intravítrea de pegaptanib sódio comprovaram a segurança e a tolerabilidade da droga, e os resultados funcionais desses estudos iniciais foram promissores, pois $26 \%$ dos pacientes tiveram melhora da acuidade visual em até seis meses de seguimento $^{(32-33)}$. Em um ensaio clínico fase 3, randomizado, duplo cego, em 117 centros especializados em doenças da retina, a eficácia clínica do pegaptanib sódio foi verificada. Os 1.208 pacientes com DMRI exudativa foram randomizados para receber pegaptanib $(0,3 \mathrm{mg}, 1,0 \mathrm{mg}$, ou 3,0 mg), ou uma injeção placebo subconjuntival controle a cada 6 semanas por 48 semanas, até uma fase de segunda randomização na qüinquagésima quarta semana. $\mathrm{O}$ tratamento foi combinado com o chamado tratamento "usual", o PDT, quando indicado. O objetivo primário do estudo foi a diminuição da chance de perda da visão ( $\geq 3$ linhas). No grupo de $0,3 \mathrm{mg}, 30 \%$ dos pacientes tratados com pegaptanib sódio perderam mais do que três linhas de visão em comparação com $45 \%$ do grupo de injeção subconjunctival de placebo $(\mathrm{p}<0,0001)$ (Figura 2). Os investigadores relataram que pegaptanib sódio foi razoavelmente eficaz em todas as doses testadas e para todos os tipos

\begin{tabular}{|c|c|c|c|c|}
\hline & Pegaptanib & Ranibizumab & Bevacizumab & VEGF-trap \\
\hline Peso molecular (kDa) & 20 & 58 & 149 & \\
\hline Tipo de proteína & Aptâmero RNA & $\begin{array}{l}\text { Fragmento de anti- } \\
\text { corpo recombinante }\end{array}$ & $\begin{array}{l}\text { Anticorpo IgG1 mono- } \\
\text { clonal recombinante }\end{array}$ & \\
\hline $\begin{array}{l}\text { Origem da seqüência } \\
\text { de aminoácidos }\end{array}$ & Tecnologia Selex & Humanos/murinos & Humanos/murinos & $\begin{array}{l}\text { Proteína fusional de partes do } \\
\text { receptor VEGFR1 e VEGFR2 } \\
\text { com porção Fc de G1 }\end{array}$ \\
\hline Meia-vida (dias) & 10 & 3 & $13-21$ & Humanos \\
\hline Via de administração & Intravítrea & Intravítrea & Endovenosa/Intravítrea & \\
\hline Ação antiangiogênica & Bloqueio VEGF ${ }_{165}$ & $\begin{array}{l}\text { Bloqueio todas } \\
\text { isoformas VEGF }\end{array}$ & $\begin{array}{l}\text { Bloqueio todas } \\
\text { isoformas VEGF }\end{array}$ & Intravítrea \\
\hline $\begin{array}{l}\text { Estágio de pesquisa } \\
\text { clínica }\end{array}$ & $\begin{array}{l}\text { Ensaio clínico fase } 3 \text {, } \\
\text { em continuação }\end{array}$ & $\begin{array}{l}\text { Ensaio clínico fase } 3 \text {, } \\
\text { em continuação }\end{array}$ & Ensaio clínico fase 1 & $\begin{array}{l}\text { Bloqueio todas isoformas } \\
\text { VEGF e PIGF }\end{array}$ \\
\hline FDA-status & Aprovado & Em avaliação & Em avaliação & $\begin{array}{l}\text { Ensaio clínico fase } 1 \\
\text { em avaliação }\end{array}$ \\
\hline Complicações & $\begin{array}{l}\text { Dor, ceratite, aumento da } \\
\text { PIO, inflamação câmara } \\
\text { anterior, catarata, edema } \\
\text { de córnea, endoftalmite, DR }\end{array}$ & $\begin{array}{l}\text { Inflamação } \\
\text { intra-ocular, } \\
\text { perivasculite }\end{array}$ & $\begin{array}{l}\text { Eventos trombo-embólicos, } \\
\text { hipertensão, proteinúria, e } \\
\text { diminuição de poder de } \\
\text { cicatrização após cirurgias }\end{array}$ & Desconhecidos \\
\hline
\end{tabular}




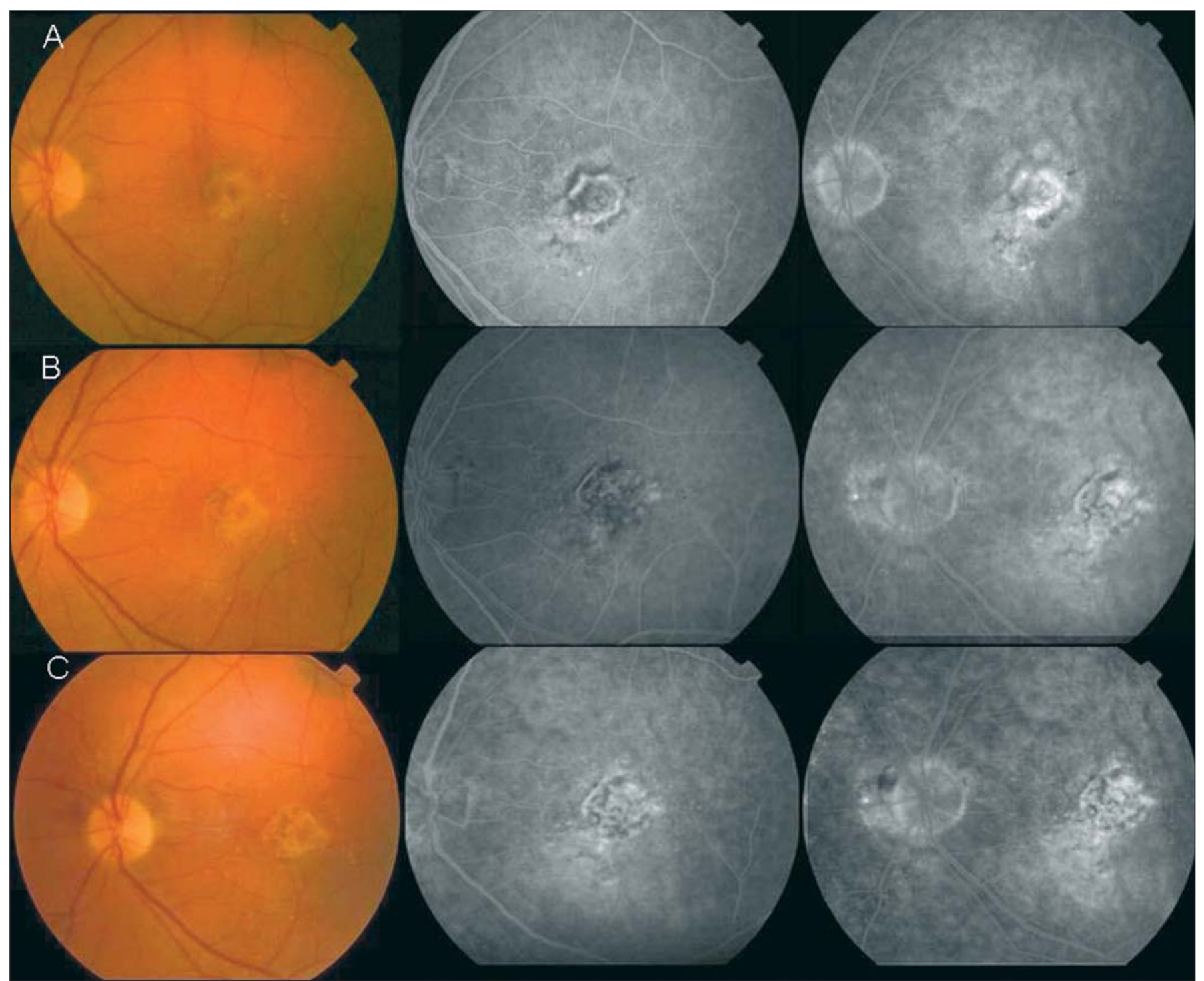

Figura 2 - Paciente com MNSR predominantemente clássica tratado com injeções intravítreas de Pegaptanib sódio. A: Retinografia e angiografia fluoresceínica $O E$ pré-tratamento. A acuidade visual está em 20/320; B: Retinografia e angiografia fluoresceínica $O E$ um ano após início do tratamento. A acuidade visual melhorou para 20/80; C: Retinografia e angiografia fluoresceínica OE dois anos após início do tratamento. Houve diminuição do vazamento na angiografia fluoresceínica nas fases tardias. A acuidade visual melhorou para 20/50.

de composições de lesão MNSR ${ }^{(34)}$. Paradoxicalmente, o grupo de alta dose de pegaptanib sódio (3 mg) não apresentou diferença estatisticamente significante do grupo controle. Um trabalho recente evidenciou maior eficácia com injeção precoce, ou seja, em casos iniciais, de pegaptanib sódio para DMRIneovascular ${ }^{(35)}$. Há a preocupação que a inibição de VEGF na circulação sistêmica poderia levar ao aumento da incidência de acidentes cardio-vasculares. No entanto, o aptâmero pegaptanib injetado intravítreo foi descrito pelo órgão "FDA" (Food and Drug Admnistration) norte-americano ser uma droga segura no ensaio clínico de larga escala. Ao custo de US\$ 995,00 por injeção, o primeiro aptâmero para uso clínico na história da medicina foi aprovado em dezembro de 2004 pelo FDA para tratamento da forma exsudativa de DMRI.

\section{Ranibizumab - RhuFab V2 (Lucentis $\left.{ }^{\circledR}\right)$}

Ranibizumab (rhuFab V2, Lucentis; Genentech, São Francisco, CA, USA) é o fragmento Fab (a porção que se liga ao antígeno) do anticorpo monoclonal anti-VEGF humanizado bevacizumab. Este anticorpo recombinante consiste em duas partes: uma seqüência humana não ligante que confere ao anticorpo menor antigenicidade; e um epitopo ligante de alta afinidade derivado de camundongo que serve para ligação ao antígeno $^{(36)}$. Ranibizumab liga-se a todas as isoformas de VEGF-A e inibe a interação entre o peptídeo VEGF e seus receptores ${ }^{(37)}$. A fragmentação do anticorpo anti-VEGF foi necessária para redução do peso molecular de 148 kDa da forma natural do anticorpo monoclonal. Com um peso molecular de 
$58 \mathrm{kDa}$, ranibizumab atinge níveis significativos no espaço sub-retiniano e coroideano.

As propriedades farmacocinéticas de ranibizumab para inibição de MNSR motivaram estudos pré-clínicos de eficácia e segurança. A injeção intravítrea de 500 microgramas de ranibizumab administrada a cada duas semanas em um modelo de MNSR induzido por laser em macacos demonstrou nenhum efeito tóxico severo e preveniu a formação de MNSR. No entanto, inflamação transitória na câmara anterior ocorreu em todos os olhos nas primeiras horas após o tratamento ${ }^{(38)}$. Um estudo de tolerabilidade em humanos feito por Rosenfeld et al. investigou a administração de doses variadas entre 50 a 2000 microgramas intravítreo de ranibizumab. Concluiu-se que a dose máxima tolerada intravítrea é de 500 microgramas, uma vez que em doses acima de 1000 microgramas, inflamação intra-ocular significante foi observada ${ }^{(39)}$. O efeito adverso mais freqüentemente observado nestes estudos pré-clínicos de ranibizumab foi uma perivasculite com o surgimento de anticorpos neutralizantes do fragmento Fab. A meia-vida intravítrea de ranibizumab foi de aproximadamente de 2 a 4 dias (Tabela 1). Um estudo fase 1 comparando injeções intravítreas mensais de ranibizumab com PDT demonstrou melhora da acuidade visual em $26 \%$ dos pacientes após 3 meses do estudo. Em um ensaio clínico fase 2, os pacientes foram randomizados em "tratamento usual" ou seja PDT, ou injeções intravítreas de 300 microgramas ou 500 microgramas a cada 28 dias em 4 doses. O tratamento com ranibizumab foi associado a poucos efeitos colaterais com exceção da inflamação ocular transitória como evidenciado em estudos pré-clínicos. A acuidade visual melhorou em média 12 letras no grupo de ranibizumab 300 microgramas em comparação com o grupo controle após 7 meses de seguimento. A empresa Genentech anunciou em 23 de maio de 2005 que o ensaio clínico fase 3 da droga ranibizumab atingiu a meta de eficácia primária, pois aproximadamente $95 \%$ dos pacientes mantiveram ou melhoraram a acuidade visual após 1 ano em comparação com apenas $62 \%$ do grupo controle. Este estudo fase 3 continua em dois braços, um para comparação com o PDT, e o outro para investigar sobre a possibilidade de aplicação da droga intravítrea num período maior que 1 mês.

\section{Bevacizumab (Avastin $\left.{ }^{\circledR}\right)$}

Bevacizumab (Avastin ${ }^{\circledR}$, Genentech, São Francisco, CA, USA) é um anticorpo IgG1 monoclonal humanizado recombinante de peso molecular de $149 \mathrm{kDa}^{(40-41)}$. A droga é composta em $93 \%$ por uma imunoglobulina $\mathrm{G}$ humana padrão, enquanto que $7 \%$ dela compreendem resíduos ligantes de VEGF produzidos de anticorpos neutralizantes de camundongos ${ }^{(42)}$. Estudos in vivo e in vitro mostraram que bevacizumab bloqueia as atividades biológicas do VEGF-A, pois não permite que qualquer isoforma de VEGF-A se ligue aos seus receptores VEGFR-1 e VEGFR-2. Com uma dose sistêmica de 3-10 mg/kg, o bevacizumab demonstra uma farmacocinética linear com um volume de distribuição limitado (Tabela 1). A droga tem um baixo "clearance" e tem uma meia-vida sistêmica de 13 a 21 dias. Altos níveis de VEGF foram correlacionados com pior prognóstico e aumento do risco de metástase, pois neoplasias malignas necessitam de neovasos para seu crescimento. Portanto, a atividade antiangiogênica de bevacizumab tem sido intensamente estudada no tratamento de pacientes com câncer. Estudos em animais demonstraram que a administração sistêmica de bevacizumab produziu a supressão e estabilização de múltiplas linhas tumorais ${ }^{(43-44)}$. Em fevereiro de 2004, o FDA aprovou o uso clínico de bevacizumab em combinação com outros quimioterápicos como agente de primeira linha no tratamento de câncer coloretal metastático e estão em andamento ensaios clínicos fase 3 para outros tipos de câncer como pulmonar, renal e de mama.

Em 1996 a droga bevacizumab foi investigada intravítrea em olhos de macacos ${ }^{(45)}$. Outro estudo por Tolentino et al. demonstrou que bevacizumab conjugado com fluoresceína vazou em MNSR criada por laser em macacos após administração sistêmica do anticorpo ${ }^{(46)}$. Um pequeno estudo prospectivo recente em nove pacientes recebendo 2 ou 3 infusões endovenosas de bevacizumab na dose de $5 \mathrm{mg} / \mathrm{kg}$ resultou em melhora da acuidade visual e restabelecimento da espessura retiniana normal no curto de período de 12 semanas de seguimento $^{(37)}$. O tratamento com bevacizumab sistêmico poderia ser indicado nos casos cuja injeção intra-ocular de medicamento como pegaptanib ou ranibizumab esteja contraindicada. Outras vantagens consistem na penetração direta sangue-MNSR, e maior meia-vida da droga em comparação com os outros tratamentos. Algumas das desvantagens de bevacizumab endovenoso incluem o risco de eventos tromboembólicos, hipertensão, proteinúria, e diminuição de poder de cicatrização após cirurgias.

Em julho de 2005 Rosenfeld et al. apresentaram surpreendentes resultados em um pequeno grupo de pacientes com MNSR e DRMI tratados com injeção intravítrea com 1,25 mg de bevacizumab (Congresso da American Society of Retina Specialists, Julho, 2005, Montreal, Canadá). Houve importante regressão clínica da MNSR em humanos, resultados estes confirmados pelo OCT. Uma grande vantagem de bevacizumab intravítrea é o custo inferior sobre outros medicamentos como Macugen e Lucentis. Em um simpósio recentemente organizado sobre Avastin, a única complicação relatada foi hemorragia vítrea em um paciente diabético (Simpósio Avastin, Curso Retina e Trauma, Escola Paulista de Medicina, 1 de dezembro de 2005), e nós tivemos um caso de ruptura do EPR em paciente com membrana oculta após injeção de bevacuzumab intravítreo (dados não publicados). Para responder a questão da segurança de bevacizumab intravítrea, Fung e colaboradores organizaram em outubro de 2005 um questionário "online" para relato de complicações com este medicamento por especialistas em retina (https://www.formrouter.net /forms@PACEA/AvastinSafetySurvey05_A.aspx), mas os resultados ainda não foram liberados até 31 de dezembro de 2005. A impressionante eficácia desta droga associado ao seu menor custo tem um impacto mundial enorme, e nos próximos anos pacientes com doenças de retina neovasculares incluindo DMRI serão muito beneficiados. 


\section{VEGF-trap}

VEGF-trap é uma proteína fusional que consiste de partes do receptor VEGFR1 e VEGFR2 do VEGF humano. Essas duas partículas foram fundidas com uma porção Fc da imunoglobulina humana IgG1. O VEGF-trap liga-se com alta afinidade e inativa a molécula de VEGF na circulação e nos tecidos. Em contraste com os anticorpos monoclonais humanizados, o VEGF-trap contém somente seqüências de aminoácidos humanos. Esta proteína tem uma capacidade de ligar-se a VEGF cem vezes mais do que anticorpos monoclonais, portanto menores doses são necessárias para a mesma eficácia. Além de inibir a glicoproteína VEGF, o VEGF-trap bloqueia também o mediador pró-angiogênico PIGF. Um estudo pré-clínico realizado em rodentes provou o potencial uso de VEGF-trap em MNSR ${ }^{(47)}$. Nesse estudo, VEGF-trap foi injetada subcutânea e intra-ocular em dois grupos. Em ambos os grupos houve diminuição no surgimento de neovascularização sub-retiniana em modelos rodentes. Pesquisa inicial em humanos demonstrou o VEGFtrap ter atividade antiangiogênica em tumores sólidos ou linfoma $^{(48)}$. Os pesquisadores não evidenciaram efeitos adversos com esse tipo de tratamento. A empresa Regeneron farmaceuticals anunciou em maio de 2005 o início de um ensaio clínico fase 1 com injeção intra-ocular de VEGF-trap em pacientes com DMRI-neovascular.

\section{Squalamina}

Nos últimos anos inúmeras substâncias como peptídeos, lipídeos e alcalóides com propriedades antibióticas e corticosteróides foram isolados em diversas espécies de animais. Uma dessas substâncias foi encontrada em extratos estomacais do tubarão da espécie Squalus acanthias, sendo denominada por este motivo squalamina ${ }^{(49)}$. A squalamina é um aminoesterol esteroidal de peso molecular de 628 Da, cationica, solúvel em água, com potentes propriedades antimicrobianas ${ }^{(50)}$. A atividade antiangiogênica do aminosterol squalamina está relacionada à sua potente propriedade como inibidor de migração e proliferação de células endoteliais ${ }^{(51)}$. A droga promove o bloqueio no receptor NHE-3, que é um receptor da bomba de sódio-potássio e de cálcio. O bloqueio do receptor NHE-3 induz a alteração de motilidade, crescimento, pH, e volume celular das células endoteliais com conseqüente inibição da angiogênese. Pesquisas em animais e iniciais em humanos evidenciaram o potencial da squalamina no tratamento de neoplasia maligna avançada ${ }^{(52-53)}$ (Tabela 2).

Alguns estudos pré-clínicos comprovaram o efeito antiangiogênico da squalamina na neovascularização ocular. A injeção sistêmica do aminoesterol squalamina levou a inibição do surgimento de neovascularização na íris em um modelo experimental de neovascularização em macacos ${ }^{(54)}$. Em um modelo de retinopatia da prematuridade em camundongos, a injeção intraperitonial de squalamina na dose de $40 \mathrm{mg} / \mathrm{kg}$ bloqueou a neovascularização retiniana significativamente ${ }^{(55)}$. Em um modelo de ratos de MNSR induzida por laser houve uma redução no aparecimento das lesões neovasculares coroideanas no grupo tratado com injeção intraperitonial com $5 \mathrm{mg} / \mathrm{kg}$ da droga ${ }^{(56)}$. Em agosto de 2002, a Corporação Genaera iniciou de ensaios clínicos fase 1 e 2 com a administração endovenosa de squalamina para pacientes com DMRI exsudativa. Os resultados dos estudos fase 2 demonstraram que todos os indivíduos que receberam $40 \mathrm{mg}$ endovenosa da squalamina tiveram a acuidade visual estável ou melhor por até 4 meses. Em outubro de 2004, Genaera recebeu um status "fast track" do FDA, e em 2005 um grande ensaio clínico fase 3 para avaliação deste agente foi iniciado.

\section{Acetato de anecortave}

Acetato de anecortave (Alcon Research Ltd., Fort Worth, Texas, USA) é uma droga derivada do cortisol sinteticamente e seu efeito antiangiogênico deve-se a modificações bioquímicas permanentes na estrutura molecular ${ }^{(57)}$. Uma ligação química dupla na posição C9-11 e a remoção do grupo $11-\beta$ hidroxil da molécula resultaram em um cortiseno sem bioatividade corticosteróide. Acetato de anecortave inibe a neovascularização primariamente pela redução da síntese de duas enzimas proteolíticas, ativador de plasminogênio uroquinaselike e matrix metaloproteinase-3, fundamentais para a migração

\begin{tabular}{|llll|}
\hline \multicolumn{2}{|c|}{ Tabela 2. Propriedades químicas, farmacocinética, e mecanismos de ação dos principais esteróides e derivados antiangiogênicos } \\
& Acetato de triancinolona & Acetato de anecortave & Squalamina \\
Fórmula molecular & $\mathrm{C}_{24} \mathrm{H}_{31} \mathrm{FO}_{6}$ & $\mathrm{C}_{23} \mathrm{H}_{30} \mathrm{O}_{5}$ & $\mathrm{C}_{34} \mathrm{H}_{65} \mathrm{~N}_{3} \mathrm{O}_{5} \mathrm{~S}-\mathrm{xC}_{3} \mathrm{H}_{6} \mathrm{O}_{3}-\mathrm{yH}_{2} \mathrm{O}$ \\
Peso molecular (daltons) & 434 & 386 & 627 \\
Origem & $\mathrm{Sintética}$ & $\mathrm{Sintética}$ & Derivada de animal - tubarão \\
Efeito corticosteróide & $\mathrm{Sim}$ & $\mathrm{Não}$ & $\mathrm{Sim}$ \\
Meia-vida intravítrea & 18 & n.a. & n.a. \\
Via de administração & Intravítrea/Subtenoniana & Justa-escleral & Endovenosa \\
Mecanismo de ação & Altera a expressão de moléculas & Redução da síntese de duas & Bloqueio receptor NHE-3 - \\
antiangiogênica & de adesão inflamatória e a permea- & enzimas proteolíticas da & alteração de motilidade, cresci- \\
& bilidade de células endoteliais & migração de células endoteliais & mento das células endoteliais \\
Estágio de pesquisa clínica & Ensaio clínico fase 1 e 2 & Ensaio clínico fase 3 & Ensaio clínico fase 3 \\
Status no FDA & Aprovada para uso sistêmico & Em avaliação & Em avaliação \\
Principais complicações & Catarata, glaucoma, & Hiperemia ocular, prurido & Fadiga, hepatotoxicidade \\
& endoftalmite, DR & & (em alta concentração) \\
\hline
\end{tabular}


das células endoteliais vasculares. Portanto, há prevenção da migração destas células de maneira independente do estímulo angiogênico pré-existente ${ }^{(58)}$ (Tabela 2).

Aplicada topicamente, intravítrea, ou peri-orbitária, o acetato de anecortave suprimiu a neovascularização patológica em vários modelos animais: neovascularização induzida por lipopolisacarídeos e fatores de crescimento fibroblástico básico, retinopatia induzida por oxigênio e em tumores intra-oculares altamente vascularizados ${ }^{(59-60)}$. Dados farmacocinéticas pré-clínicos não publicados concluíram que acetato de anecortave possui maior eficácia quando administrado na superfície justa-escleral. Esta via de administração tem a vantagem de evitar complicações como descolamento de retina e endoftalmite. Após mais de 1.200 aplicações de acetato de anecortave em diferentes fases de ensaios clínicos e doses poucos efeitos adversos foram verificados. Os resultados do primeiro ensaio clínico em larga escala do acetato de anecortave revelaram melhora estatisticamente significante da acuidade visual no grupo tratado com $15 \mathrm{mg}$ de acetato de anecortave em comparação com placebo $(\mathrm{p}=0,003)$. No entanto, $88 \%$ dos pacientes tratados com a droga mantiveram a acuidade visual em comparação com $70 \%$ do grupo placebo e esses resultados não foram estatisticamente significantes $(p=0,080)^{(61-62)}$. No fim do primeiro ano do estudo, $15 \mathrm{mg}$ de acetato de anecortave administrado em intervalos de 6 meses foi estatisticamente superior ao placebo para 3 métodos de eficácia clinica: estabilização da visão, prevenção de perda visual severa, e variação média da acuidade visual. A empresa especula que a causa do insucesso relativo neste tratamento seria o alto índice de refluxo do medicamento. Uma cânula especial curva e um dispositivo de contrapressão para evitar refluxo da droga foram desenvolvidos para a aplicação da droga justa-escleral, e novos ensaios clínicos foram iniciados. Paralelamente, iniciou-se um importantíssimo ensaio clínico em que se refere à forma seca de DMRI. Este estudo consiste na aplicação justaescleral de acetato de anecortave em pacientes com alto risco de desenvolver MSNR, ou seja, aqueles com drusas mole em um olho e cicatriz disciforme no olho contralateral ${ }^{(63)}$. Aguarda-se para um futuro próximo a informação sobre a exata indicação do acetato de anecortave na profilaxia e tratamento da MNSR na DRMI.

\section{Acetato de triancinolona}

Acetato de triancinolona (AT) é um corticosteróide derivado mais potente do que a forma nativa da triancinolona com peso molecular de $434 \mathrm{Da}$. O AT tem atividade antiinflamatória de 4 a 6 vezes mais potente do que a forma triancinolona pura. No fim da década de 70, Machemer et al. em estudo experimentais e clínicos propuseram que corticosteróides poderiam ser injetados intra-ocular para tratamento de várias doenças exsudativas e proliferativas ${ }^{(64-65)}$. Devido ao fato que corticosteróides líquidos dissolvem-se em 24 horas do tecido intraocular, idealizou-se que os corticosteróides cristalinos, como o AT, poderiam permanecer vários meses dentro do olho e ter uma ação prolongada ${ }^{(66)}$. Somente então no fim da década de
90 foi difundido o uso clínico AT no tratamento de edemas maculares de diferentes causas (diabéticos, pseudofácicos, telangiectásicos, e retinopatia radioativa) ${ }^{(67)}$. E conseqüentemente os efeitos adversos foram identificados, e estes incluem catarata, glaucoma e endoftalmite (Tabela 2).

Trabalhos experimentais in vitro e in vivo em animais revelaram a potencial atividade antiangiogênica do corticosteróide AT. Em um modelo de neovascularização de retinopatia da prematuridade em camundongos, a injeção intravítrea de AT gerou uma significante redução na neovascularização retiniana ${ }^{(67)}$. Concluiu-se recentemente que a AT também altere a expressão de moléculas de adesão inflamatória e a permeabilidade de células endoteliais coroideanas ${ }^{(68)}$. O mecanismo angiostático de AT na regressão da MNSR pode estar relacionado a um efeito direto pela atenuação na migração e ativação de células inflamatórias que contribuem na liberação de citocinas na MNSR. Adicionalmente, TA pode inibir a expressão de VEGF, b-FGF, e outros mediadores bioquímicos pró-angiogênicos ${ }^{(69-70)}$.

A primeira descrição na literatura do uso clínico de AT para DMRI foi realizada em 1995 por Penfold et al. num estudo piloto em uma pequena série de pacientes ${ }^{(71)}$. Após isso, Challa et al. em 1998 testaram a toxicidade e eficácia de $4 \mathrm{mg}$ de AT intravítrea num período de 18 meses de seguimento em pacientes com MNSR ${ }^{(72)}$. Posteriormente, um estudo prospectivo comparou injeção de $4 \mathrm{mg}$ de AT em 27 pacientes com grupo controle, e houve melhora estatisticamente significante da acuidade visual no grupo tratado após 6 meses $^{(73)}$. Um estudo prospectivo avaliou o efeito de $25 \mathrm{mg}$ intravítreo de AT em 71 olhos com DMRI-neovascular ${ }^{(74)}$. Houve uma leve melhora da acuidade visual média, mas esse efeito foi mais intenso nos primeiros três meses após tratamento com progressiva redução no período subseqüente. Devido ao efeito progressivo da MNSR em DMRI e o efeito temporário da AT de 3 a 6 meses, uma dose única de AT intravítrea provavelmente não é suficiente para inibir a angiogênese na DMRI. Uma outra abordagem recentemente publicada foi a combinação de AT com PDT ${ }^{(75)}$. Quando usadas concomitantemente, esta combinação reduziu a necessidade de re-tratamento com PDT, e especula-se que AT reduza inflamação e lesão da coróide relacionada com o PDT. Os resultados dos estudos com esta combinação são de difícil interpretação, pois não houve um padrão de tratamento e uma homogeneização entre os vários tipos de MNSR e de tratamento. No entanto, vários estudos em larga escala prospectivos e randomizados estão em andamento para avaliação de PDT combinada com AT no tratamento de DMRI exsudativa.

\section{Moléculas de RNA-interference}

Moléculas de RNA-interference (RNAi) são construídas por uma técnica gênica, o resultado é a formação de RNA de dupla hélice desenvolvidos para atingir um RNA mensageiro (mRNA) especifico. RNAi destroem o mRNA silenciando a expressão de um gene alvo. O "small” iRNA (siRNA) contém 21 nucleotídeos em dupla hélice que inibe a transcrição e 
produção de mRNA patológico como VEGF. Uma molécula de siRNA pode destruir centenas de mRNA, e o resultado é a supressão de milhares de proteínas de VEGF. Ao invés de antagonizar o VEGF após ele ser produzido, o siRNA pode suprimir a produção de VEGF em sua totalidade. Em estudos pré-clínicos o siRNA direcionado para VEGF inibiu fortemente a expressão de VEGF em modelos murinos, e inibiu neovascularização induzida por laser em camundongos e macacos sem sinais de toxicidade ${ }^{(76)}$. Em setembro de 2004 a empresa "Acuity Pharmaceuticals" (Acuity Pharmaceuticals, Philadelphia, PA) iniciou um estudo fase I "Cand-5 therapy" com siRNA anti-VEGF para pacientes com DRMI exsudativa. A empresa Sirna Therapeutics desenvolveu um siRNA modificado (Sirna027) que age especificamente no receptor VEGFR-1, e em novembro de 2004 um estudo fase 1 foi iniciado em 30 pacientes com MNSR, com resultados preliminares a serem liberados em 2006. Um problema deste tipo de tratamento com siRNA será o seu alto custo, uma vez que tecnologia avançadíssima foi necessária para seu desenvolvimento ${ }^{(77)}$.

\section{CONSIDERAÇÕES FINAIS}

O conhecimento da fisiopatogenia molecular da MNSR e da família de peptídeos VEGF facilitou o desenvolvimento de diferentes tipos de tratamentos biológicos para a forma neovascular de DMRI. A primeira droga liberada pelo FDA, o pegaptanib sódio teve eficácia semelhante ao PDT em reduzir a chance de perda visual em todos os subtipos de MNSR. Os resultados iniciais de bevacizumab intravítreo foram formidáveis com mínimas complicações associadas. Há uma grande expectativa para conhecer o desfecho dos ensaios clínicos das novas drogas antiangiogênicas, e a definição da indicação específica de cada tipo de cada droga. E no futuro provavelmente, especula-se que iniciar-se-ão ensaios clínicos com tratamentos biológicos antiangiogênicos combinados. Espera-se também para um futuro a utilização da modalidade farmacológica também na prevenção da formação das formas avançadas de DMRI, e a primeira droga a ser investigada para esta finalidade é o acetato de anecortave. A abordagem farmacológica antiangiogênica abre um leque enorme de perspectivas no futuro para nossos pacientes.

\section{AGRADECIMENTOS}

Os autores agradecem a empresa Eyetech por fornecerem as figuras $2 \mathrm{~A}, \mathrm{~B}$, e $\mathrm{C}$ para este artigo.

\section{ABSTRACT}

Age-related macular degeneration (ARMD) remains a leading cause of blindness in the western world. Several clinical forms of the disease are recognized, whereas choroidal neovascularization $(\mathrm{CNV})$ represents an important manifestation suitable for treatment. The treatment of CNV has been a major focus of research in the past decades, and the first evidence-based established therapy was laser photocoagulation, which reduces the risk of visual loss in extrafoveal lesions. In the late 90's photodynamic therapy has been established as an efficient method for the treatment of predominantly classic and occult CNV. Additional therapies such as macular translocation, submacular surgery, and indocyanine-mediated prothrombosis are currently under investigation in large-scale clinical trials. Molecular biology has recently provided a better comprehension of the pathogenesis of ARMD, and vascular endothelial growth factor (VEGF) was recognized as key mediator in the angiogenesis of CNV-formation. Therefore, the pharmacological approach rose as a key research area to treat $\mathrm{CNV}$. The first FDA-approved agent for CNV-therapy is aptamer pegaptanib sodium $\left(\right.$ Macugen $^{\circledR}$ ), which inactivates the key angiogenic isoform $\mathrm{VEGF}_{165}$. Additional VEGF-blockers such as ranibizumab RhuFab V2 (Lucentis ${ }^{\circledR}$ ) and bevacizumab $\left(\right.$ Avastin $\left.^{\circledR}\right)$ are under evaluation in major clinical studies. Impressive results of intravitreal bevacizumab were released recently. Moreover, the steroid-derived anecortave acetate as well as the corticosteroid triamcinolone acetate have been proposed as methods for treatment of wet-ARMD. This paper presents the rationale and principles of the pharmacologic antiangiogenic therapy for CNV in ARMD.

Keywords: Macular degeneration; Retinal neovascularization/drug therapy; Vascular endothelial growth factor; Angiogenesis inhibitors

\section{REFERÊNCIAS}

1. Medina NH, Barros OM, Muñoz EH, Magdaleno RL, Barros AJD, Ramos LR. Morbidade ocular em idosos da cidade de São Paulo - SP, Brasil. Arq Bras Oftalmol. 1993;56(5):276-8, 281-3.

2. Santos LPF, Diniz JR, Leão ACS, Sena MF. Degeneração macular relacionada à idade: prevalência e fatores de risco em dois centros oftalmológicos de referência em Pernambuco. Arq Bras Oftalmol. 2005;68(2):229-33.

3. Nehemy M, Passos E, Campos C, Rodrigues RP, Nehemy DB. Indocianina verde como adjuvante da termoterapia transpupilar em membrana neovascular subretiniana secundária à degeneração macular relacionada à idade. Rev Bras Oftalmol. 2001;60(4):251-9.

4. Oliveira Neto H, Bordon AF, Farah ME, Breuer M, Uno F. Estudo e classificação das membranas neovasculares subretinianas na degeneração macular relacionada à idade pela angiografia digital com indocianina verde. Arq Bras Oftalmol. 1998;61(3):312-22.

5. Casella AMB, Taba KE, Ramos A, Farah ME. Indocianina verde vídeo-angiografia e oftalmoscopia de rastreamento a laser em neovascularização subretiniana. Arq Bras Oftalmol. 1998;61(2):230-2.

6. Ávila MP. Subretinal neovascularization: contribution on angiographic and therapeutic study of patients with age-related macular degeneration [dissertation]. Belo Horizonte: Universidade Federal de Minas Gerais; 1997.

7. Farah ME, Oshima A, Costa RA, Sallum JF. Degeneração macular relacionada à idade: modalidades terapêuticas. Arq Bras Oftalmol. 2001;64(6):583-8.

8. Argon laser photocoagulation for neovascular maculopathy. Three-year results from randomized clinical trials. Macular Photocoagulation Study Group. Arch Ophthalmol. 1986;104(5):694-701.

9. Farah ME, Cardillo JA, Luzardo AC, Calucci D, Williams GA, Costa RA. Indocyanine green mediated photothrombosis for the management of predominantly classic choroidal neovascularization caused by age related macular degeneration. Br J Ophthalmol. 2004;88(8):1055-9. 
10. Sattler H. [Ueber den feineren Bau der Chorioidea des Menschen nebst Beitragen zur pathologischen und vergleichenden Anatomie der Aderhaut]. Albrecht von Graefe's Archiv fur Ophthalmologie. 1876; 22(2):1-100. German.

11. Grossniklaus HE, Green WR. Choroidal neovascularization. Am J Ophthalmol. 2004;137(3):496-503.

12. Grunwald JE, Metelitsina TI, Dupont JC, Ying GS, Maguire MG. Reduced foveolar choroidal blood flow in eyes with increasing AMD severity. Invest Ophthalmol Vis Sci. 2005;46(3):1033-8.

13. Penfold PL. Inflammation and age-related macular degeneration. JAMA. 2004; 292(1):43; author reply 43.

14. Penfold PL, Madigan MC, Gillies MC, Provis JM. Immunological and aetiological aspects of macular degeneration. Prog Retin Eye Res. 2001;20(3):385-414.

15. Kijlstra A, La Heij E, Hendrikse F. Immunological factors in the pathogenesis and treatment of age-related macular degeneration. Ocul Immunol Inflamm. 2005;13(1):3-11.

16. Grossniklaus HE, Martinez JA, Brown VB, Lambert HM, Sternberg P Jr, Capone A Jr, et al. Immunohistochemical and histochemical properties of surgically excised subretinal neovascular membranes in age-related macular degeneration. Am J Ophthalmol. 1992;114(4):464-72.

17. Winkler BS, Boulton ME, Gottsch JD, Sternberg P. Oxidative damage and age-related macular degeneration. Mol Vis. 1999;5:32.

18. Kuroki M, Voest EE, Amano S, Beerepoot LV, Takashima S, Tolentino M, et al. Reactive oxygen intermediates increase vascular endothelial growth factor expression in vitro and in vivo. J Clin Invest. 1996;98(7):1667-75.

19. Edwards AO, Ritter R $3^{\text {rd }}$, Abel KJ, Manning A, Panhuysen C, Farrer LA. Complement factor $\mathrm{H}$ polymorphism and age-related macular degeneration. Science. 2005;308(5720):421-4.

20. Grossniklaus HE, Ling JX, Wallace TM, Dithmar S, Lawson DH, Cohen C, et al. Macrophage and retinal pigment epithelium expression of angiogenic cytokines in choroidal neovascularization. Mol Vis. 2002;8:119-26.

21. Senger DR, Galli SJ, Dvorak AM, Perruzzi CA, Harvey VS, Dvorak HF. Tumor cells secrete a vascular permeability factor that promotes accumulation of ascites fluid. Science. 1983;219(4587):983-5.

22. Klagsbrun M, D'Amore PA. Vascular endothelial growth factor and its receptors. Cytokine Growth Factor Rev. 1996;7(3):259-70. Review.

23. Witmer AN, Vrensen GF, Van Noorden CJ, Schlingemann RO. Vascular endothelial growth factors and angiogenesis in eye disease. Prog Retin Eye Res. 2003;22(1):1-29.

24. Carmeliet P, Jain RK. Angiogenesis in cancer and other diseases. Nature. 2000; 407(6801):249-57.

25. Shweiki D, Itin A, Soffer D, Keshet E. Vascular endothelial growth factor induced by hypoxia may mediate hypoxia-initiated angiogenesis. Nature. 1992; 359(6398):843-5.

26. Jin KL, Mao XO, Greenberg DA. Vascular endothelial growth factor: direct neuroprotective effect in in-vitro ischemia. Proc Natl Acad Sci U S A. 2000;97 (18):10242-7.

27. Adamis AP, Shima DT, Tolentino MJ, Gragoudas ES, Ferrara N, Folkman J, et al. Inhibition of vascular endothelial growth factor prevents retinal ischemiaassociated iris neovascularization in a nonhuman primate. Arch Ophthalmol. 1996;114(1):66-71.

28. Adamis AP, Miller JW, Bernal MT, D'Amico DJ, Folkman J, Yeo TK, Yeo KT. Increased vascular endothelial growth factor levels in the vitreous of eyes with proliferative diabetic retinopathy. Am J Ophthalmol. 1994;118(4):445-50.

29. Neufeld G, Cohen T, Gengrinovitch S, Poltorak Z. Vascular endothelial growth factor (VEGF) and its receptors. FASEB J. 1999;13(1):9-22. Review.

30. Moshfeghi AA, Puliafito CA. Pegaptanib sodium for the treatment of neovascular age-related macular degeneration. Expert Opin Investig Drugs. 2005;14(5): 671-82. Review.

31. van Wijngaarden P, Coster DJ, Williams KA. Inhibitors of ocular neovascularization: promises and potential problems. JAMA. 2005;293(12):1509-13.

32. Eyetech Study Group. Preclinical and phase 1A clinical evaluation of an antiVEGF pegylated aptamer (EYE001) for the treatment of exudative age-related macular degeneration. Retina. 2002;22(2):143-52.

33. Eyetech Study Group. Anti-vascular endothelial growth factor therapy for subfoveal choroidal neovascularization secondary to age-related macular degeneration: phase II study results. Ophthalmology. 2003;110(5):979-86.

34. Gragoudas ES, Adamis AP, Cunningham ET Jr, Feinsod M, Guyer DR; VEGF Inhibition Study in Ocular Neovascularization Clinical Trial Group. Pegaptanib for neovascular age-related macular degeneration. N Engl J Med. 2004;351(27):2805-16.

35. Gonzales CR; VEGF Inhibition Study in Ocular Neovascularization (V.I.S.I.O.N.) Clinical Trial Group. Enhanced efficacy associated with early treatment of neovas- cular age-related macular degeneration with pegaptanib sodium: an exploratory analysis. Retina. 2005;25(7):815-27.

36. Mordenti J, Cuthbertson RA, Ferrara N, Thomsen K, Berleau L, Licko V, et al. Comparisons of the intraocular tissue distribution, pharmacokinetics, and safety of 125I-labeled full-length and Fab antibodies in rhesus monkeys following intravitreal administration. Toxicol Pathol. 1999;27(5):536-44.

37. Michels S, Rosenfeld PJ. Treatment of neovascular age-related macular degeneration with Ranibizumab/Lucentis. Klin Monatsbl Augenheilkd. 2005; 222(6): 480-4. Id: Ger.

38. Gaudreault J, Fei D, Rusit J, Suboc P, Shiu V. Preclinical pharmacokinetics of Ranibizumab (rhuFabV2) after a single intravitreal administration. Invest Ophthalmol Vis Sci. 2005;46(2):726-33.

39. Rosenfeld PJ, Schwartz SD, Blumenkranz MS, Miller JW, Haller JA, Reimann $\mathrm{JD}$, et al. Maximum tolerated dose of a humanized anti-vascular endothelial growth factor antibody fragment for treating neovascular age-related macular degeneration. Ophthalmology. 2005;112(6):1048-53.

40. Gordon MS, Margolin K, Talpaz M, Sledge GW Jr, Holmgren E, Benjamin R, et al. Phase I safety and pharmacokinetic study of recombinant human antivascular endothelial growth factor in patients with advanced cancer. J Clin Oncol. 2001;19(3):843-50.

41. Gordon MS. Vascular endothelial growth factor as a target for antiangiogenic therapy. J Clin Oncol. 2000;18(21Suppl):45S-6S.

42. Presta LG, Chen H, O'Connor SJ, Chisholm V, Meng YG, Krummen L, et al. Humanization of an anti-vascular endothelial growth factor monoclonal antibody for the therapy of solid tumors and other disorders. Cancer Res. 1997;57(20): 4593-9.

43. Folkman J. Angiogenesis in cancer, vascular, rheumatoid and other disease. Nat Med. 1995;1(1):27-31.

44. Hampton T. Monoclonal antibody therapies shine in breast cancer clinical trials. JAMA. 2005;293(24):2985-9.

45. Adamis AP, Shima DT, Tolentino MJ, Gragoudas ES, Ferrara N, Folkman J, et al. Inhibition of vascular endothelial growth factor prevents retinal ischemiaassociated iris neovascularization in a nonhuman primate. Arch Ophthalmol. 1996;114(1):66-71.

46. Tolentino MJ, Husain D, Theodosiadis P, Gragoudas ES, Connolly E, Kahn J, et al. Angiography of fluoresceinated anti-vascular endothelial growth factor antibody and dextrans in experimental choroidal neovascularization. Arch Ophthalmol. 2000;118(1):78-84.

47. Saishin Y, Saishin Y, Takahashi K, Lima e Silva R, Hylton D, Rudge JS, et al. VEGF-TRAP (R1R2) suppresses choroidal neovascularization and VEGFinduced breakdown of the blood-retinal barrier. J Cell Physiol. 2003;195(2): 241-8

48. Konner J, Dupont J. Use of soluble recombinant decoy receptor vascular endothelial growth factor trap (VEGF Trap) to inhibit vascular endothelial growth factor activity. Clin Colorectal Cancer. 2004;(4 Suppl 2):S81-5.

49. Moore KS, Wehrli S, Roder H, Rogers M, Forrest JN Jr, McCrimmon D, Zasloff M. Squalamine: an aminosterol antibiotic from the shark. Proc Natl Acad Sci U S A. 1993;90(4):1354-8.

50. Stone R. Deja vu guides the way to new antimicrobial steroid. Science. 1993; 259(5098):1125.

51. Hao D, Hammond LA, Eckhardt SG, Patnaik A, Takimoto CH, Schwartz GH, et al. A Phase I and pharmacokinetic study of squalamine, an aminosterol angiogenesis inhibitor. Clin Cancer Res. 2003;9(7):2465-71.

52. Akhter S, Nath SK, Tse CM, Williams J, Zasloff M, Donowitz M. Squalamine, a novel cationic steroid, specifically inhibits the brush-border $\mathrm{Na}+\mathrm{H}+$ exchanger isoform NHE3. Am J Physiol. 1999;276(1 Pt 1):C136-44.

53. Sills AK Jr, Williams JI, Tyler BM, Epstein DS, Sipos EP, Davis JD, et al. Squalamine inhibits angiogenesis and solid tumor growth in vivo and perturbs embryonic vasculature. Cancer Res. 1998;58(13):2784-92.

54. Genaidy M, Kazi AA, Peyman GA, Passos-Machado E, Farahat HG, Williams JI, et al. Effect of squalamine on iris neovascularization in monkeys. Retina. 2002;22(6):772-8

55. RD, Sanders RJ, Yan Y, Zasloff M, Williams JI. Squalamine improves retinal neovascularization. Invest Ophthalmol Vis Sci. 2000;41(6):1507-12.

56. Ciulla TA, Criswell MH, Danis RP, Williams JI, McLane MP, Holroyd KJ. Squalamine lactate reduces choroidal neovascularization in a laser-injury model in the rat. Retina. 2003;23(6):808-14.

57. Vinores SA. Anecortave (Alcon laboratories). IDrugs. 2005;8(4):327-34.

58. Joussen AM, Kirchhof B. [Angiogenesis in ophthalmology]. A theme with a future. Ophthalmologe. 2003;100(4):281-3. German.

59. Clark AF, Mellon J, Li XY, Ma D, Leher H, Apte R, et al. Inhibition of intraocular tumor growth by topical application of the angiostatic steroid anecortave acetate. Invest Ophthalmol Vis Sci. 1999;40(9):2158-62. 
60. Penn JS, Rajaratnam VS, Collier RJ, Clark AF. The effect of an angiostatic steroid on neovascularization in a rat model of retinopathy of prematurity. Invest Ophthalmol Vis Sci. 2001;42(1):283-90.

61. D'Amico DJ, Goldberg MF, Hudson H, Jerdan JA, Krueger S, Luna S, Robertson SM, Russell S, Singerman L, Slakter JS, Sullivan EK, Yannuzzi L, Zilliox P; Anecortave Acetate Clinical Study Group. Anecortave acetate as monotherapy for the treatment of subfoveal lesions in patients with exudative age-related macular degeneration (AMD): interim (month 6) analysis of clinical safety and efficacy. Retina. 2003;23(1):14-23.

62. D’Amico DJ, Goldberg MF, Hudson H, Jerdan JA, Krueger DS, Luna SP, Robertson SM, Russell S, Singerman L, Slakter JS, Yannuzzi L, Zilliox P; Anecortave Acetate Clinical Study Group. Anecortave acetate as monotherapy for treatment of subfoveal neovascularization in age-related macular degeneration: twelve-month clinical outcomes. Ophthalmology. 2003;110(12):2372-83; discussion 2384-5.

63. Schachat AP. New treatments for age-related macular degeneration. Ophthalmology. 2005;112(4):531-2.

64. Machemer R, Sugita G, Tano Y. Treatment of intraocular proliferations with intravitreal steroids. Trans Am Ophthalmol Soc. 1979;77:171-80.

65. Machemer R. Five cases in which a depot steroid (hydrocortisone acetate and methylprednisolone acetate) was injected into the eye. Retina. 1996;16(2):166-7.

66. Schindler RH, Chandler D, Thresher R, Machemer R. The clearance of intravitreal triamcinolone acetonide. Am J Ophthalmol. 1982;93(4):415-7.

67. Jonas JB, Kreissig I, Kamppeter B, Degenring RF. Intravitreales Triamcinolonacetonid zur Behandlung intraokulärer ödematöser und neovaskulärer Erkrankungen. Ophthalmologe. 2004;101(2):113-20.

68. Penfold PL, Wong JG, Gyory J, Billson FA. Effects of triamcinolone acetonide on microglial morphology and quantitative expression of MHC-II in exudative age-related macular degeneration. Clin Experiment Ophthalmol. 2001;29(3): 188-92.

69. Kato A, Kimura H, Okabe K, Okabe J, Kunou N, Nozaki M, Ogura Y. Suppression of laser-induced choroidal neovascularization by posterior subtenon administration of triamcinolone acetonide. Retina. 2005;25(4):503-9.

70. Folkman J, Ingber DE. Angiostatic steroids. Method of discovery and mechanism of action. Ann Surg. 1987;206(3):374-83.

71. Penfold PL, Gyory JF, Hunyor AB, Billson FA. Exudative macular degeneration and intravitreal triamcinolone. A pilot study. Aust N Z J Ophthalmol. 1995;23(4):293-8.

72. Challa JK, Gillies MC, Penfold PL, Gyory JF, Hunyor AB, Billson FA. Exudative macular degeneration and intravitreal triamcinolone: 18 month follow up Aust N Z J Ophthalmol. 1998;26(4):277-81.

73. Danis RP, Ciulla TA, Pratt LM, Anliker W. Intravitreal triamcinolone acetonide in exudative age-related macular degeneration. Retina. 2000;20(3):244-50.

74. Jonas JB, Kreissig I, Hugger P, Sauder G, Panda-Jonas S, Degenring R. Intravitreal triamcinolone acetonide for exudative age-related macular degeneration. Br J Ophthalmol. 2003;87(4):462-8.

75. Spaide RF, Sorenson J, Maranan L. Combined photodynamic therapy with verteporfin and intravitreal triamcinolone acetonide for choroidal neovascularization. Ophthalmology. 2003;110(8):1517-25.

76. Reich SJ, Fosnot J, Kuroki A, Tang W, Yang X, Maguire AM, et al. Small interfering RNA (siRNA) targeting VEGF effectively inhibits ocular neovascularization in a mouse model. Mol Vis. 2003;9:210-6.

77. Tolentino MJ, Brucker AJ, Fosnot J, Ying GS, Wu IH, Malik G, et al. Intravitreal injection of vascular endothelial growth factor small interfering RNA inhibits growth and leakage in a nonhuman primate, laser-induced model of choroidal neovascularization. Retina. 2004;24(1):132-8. 